\title{
Asymptotics of Solution of a Boundary Value Problem for Quasilinear Non-Classical Type Differential Equation of Arbitrary Odd Order
}

\author{
Mahir M. Sabzaliev ${ }^{1^{*}}$ and Ilhama M. Sabzalieva ${ }^{1}$ \\ ${ }^{1}$ Azerbaijan State Oil and Industrial University, Az 1026, 20, Azadliq Ave, Baku, Azerbaijan.
}

Authors' contributions

This work was carried out in collaboration between both authors. Author MMS introduced the original problem. Author IMS carried out the iteration process and estimated the remainder term. The formulation of the main result was conducted by Mahir M. Sabzaliev. Both authors read and approved the final manuscript.

Article Information

DOI: 10.9734 BJMCS/2017/33704 Editor(s):

(1) Nikolaos Dimitriou Bagis, Department of Informatics and Mathematics, A $\overline{\text { ristotelian }}$ University of Thessaloniki, Greece. Reviewers:

(1) Guldem Yildiz, Omer Halisdemir University, Turkey. (2) Suleyman Cengizci, Middle East Technical University, Turkey. Complete Peer review History: http://www.sciencedomain.org/review-history/19309

\begin{abstract}
In a rectangle domain, a boundary value problem is considered for a singularly perturbed quasilinear non-classical type equation of arbitrary odd order, degenerating into a hyperbolic equation. Asymptotic expansion of the generalized solution of the problem under consideration is constructed to within any positive degree of a small parameter, and the residual term is estimated.
\end{abstract}

Keywords: Asymptotics; boundary layer function; remainder term.

2010 Mathematics Subject Classification: 35J62, 35J25, 65N99.

\footnotetext{
*Corresponding author: E-mail: sabzalievm@mail.ru;
} 


\section{Introduction and Problem Statement}

When studying numerical phenomena where there are nonuniform transitions from one physical characteristics to another ones, it is necessary to study singularly perturbed boundary value problems (see e.g. [1]-[4]). Singularly perturbed problems were first studied from different positions by A.N. Tikhonov [5], L.S. Pontryagin [6], I. Vishik and Lusternik [7], [8], Vazov [9], S.A. Lomov [10], A.M. Ilin [11], and other scientists.

Theory of singularly perturbed boundary value problems for linear partial equations was significantly developed in M.I. Vishik's and L.A. Lusternik's papers [7, 8]. After appear ance of these papers, this method was generalized both by the followers of M.I. Vishik and A.A. Lusternik, and by other researchers. However, all of studied boundary value problems were related to one of three classical types.

In $[7, \S 8]$ M.I. Vishik and L.A. Lusternik introduced the so called one-characteristic linear equations that are not classical equations. They called the $2 k+1$ odd order equation of the form $A_{1}\left(A_{2 k} u\right)+$ $B_{2 k} u=f$ one-characteristic if $A_{1}$ is a first order operator, $A_{2 k}$ is an elliptic operator of order $2 k$, while $B_{2 k}$ is any differential operator of order at most $2 k$. In the paper [7], they studied mutual degenerations of one-characteristic and elliptic equations. In this paper, in $D=\{(x, y) \mid 0 \leq x \leq 1,0 \leq y \leq 1\}$ they considered the boundary value problem

$$
\begin{gathered}
\varepsilon^{2} \frac{\partial}{\partial x}(\Delta u)-\varepsilon \Delta u+\frac{\partial u}{\partial x}+\frac{\partial u}{\partial y}+u=f(x, y), \\
\left.u\right|_{\Gamma}=0,\left.\quad \frac{\partial u}{\partial x}\right|_{x=1}=0
\end{gathered}
$$

where $\varepsilon>0$ is a small parameter, $\Delta \equiv \frac{\partial^{2}}{\partial x^{2}}+\frac{\partial^{2}}{\partial y^{2}}$ is the Laplace operator, $f(x, y)$ is the given function, $\Gamma$ is the boundary of the domain $D$. Assuming that $f(x, y)$ for $x=y$ together with its derivatives of appropriate order vanishes, they constructed only the first terms of the asymptotic solution of boundary value problem (1.1), (1.2).

In the paper [12], M.G. Javadov and M.M. Sabzaliev rejecting from the condition of vanishing of the function $f(x, y)$ for $x=y$ constructed first members of the asymptotic solution of boundary value problem $(1.1),(1.2)$ allowing for inner layers arising near $x=y$. In this paper, the complete asymptotics of the solution of a boundary value problem for equation (1.1) in the infinite strip $\Pi=\{(x, y) \mid 0 \leq x \leq 1,-\infty<y<+\infty\}$ was also constructed. Complete asymptotics of the solution of boundary value problem (1.1), (1.2) was constructed by Sabzaliev M.M. in [13].

Degeneration of an one-characteristic equation into an elliptic one was studied in the paper [7] on the following boundary value problem on a rectangle $D$ :

$$
\frac{\partial}{\partial x}(\Delta u)-\Delta u=f(x, y),\left.\quad u\right|_{\Gamma}=0,\left.\quad \frac{\partial u}{\partial x}\right|_{x=1}=0 .
$$

Complete asymptotics in a small parameter of the solution of a boundary value problem in the infinite strip $\Pi=\{(x, y) \mid 0 \leq x \leq 1,-\infty<y<+\infty\}$ for the equation

$$
\varepsilon \frac{\partial}{\partial x}(\Delta u)-\Delta u+a u=f(x, y), \quad(a=\text { const }>0)
$$

was constructed by M.M. Sabzaliev in [14]. M.M. Sabzaliev and M.E. Kerimova studied degeneration of one-characteristic equation into parabolic one. In the paper [15], in a rectangle

$D=\{(t, x) \mid 0 \leq t \leq T, 0 \leq x \leq 1\}$ they considered the following boundary value problem

$$
\varepsilon^{2} \frac{\partial}{\partial x}(\Delta u)-\varepsilon \frac{\partial^{2} u}{\partial t^{2}}+\frac{\partial u}{\partial t}-\frac{\partial^{2} u}{\partial x^{2}}+a u=f(t, x),
$$




$$
\begin{gathered}
\left.u\right|_{t=0}=0,\left.\quad u\right|_{t=T}=0,\left.\quad \frac{\partial u}{\partial t}\right|_{t=T}=0, \quad(0 \leq x \leq 1) \\
\left.u\right|_{x=0}=\left.u\right|_{x=1}=0, \quad(0 \leq t \leq T)
\end{gathered}
$$

where $\Delta \equiv \frac{\partial^{2}}{\partial t^{2}}+\frac{\partial^{2}}{\partial x^{2}}, a=$ const $>0$. Complete asymptotics of the solution of boundary value problem (1.3)-(1.5) was constructed. In the paper [16] a boundary value problem was studied for equation in the semi-infinite strip $\Pi_{+}=\{(t, x) \mid 0 \leq t \leq 1,0 \leq x<+\infty\}$. In this case boundary conditions (1.4) remain and instead of conditions in (1.5), the followings are considered: $\left.u\right|_{x=0}=0, \lim _{x \rightarrow+\infty} u=0$. A boundary value problem in the infinite strip

$$
\Pi=\{(t, x) \mid 0 \leq t \leq 1,-\infty<x<+\infty\}
$$

for equation (1.3) was considered in [17]. Here, instead of condition (1.5) the boundary conditions $\lim _{|x| \rightarrow+\infty} u=0$ are taken.

Some singularly perturbed linear equations of non-classical type were researched by Ya. Sh. Salimov and I.M. Sabzalieva in the papers [18]-[22].

The all above-mentioned studies are related to linear differential equations of non-classical type. The Vishik-Lusternik technique for constructing asymptotics in a small parameter of solutions of boundary value problems for linear and differential equations are taken to some classes of nonlinear differential equations as well. However, study of nonlinear singularly perturbed boundary value problems by this technique is accompanied by bulky calculations. In the paper [23], M.I. Vishik and L.A. Lusternik illustrated the technique for constructing nonlinear differential equations on the following boundary value problem:

$$
\varepsilon y^{\prime \prime}+\varphi(x, y) y^{\prime}-\psi(x, y), y(0)=A, y(1)=B .
$$

Asymptotic of the solution of this problem in powers of parameters $A$ was studied by V. Vazov.

In [24] Su Yui Chen constructed asymptotics in small parameter of the solution of a mixed problem for the quasilinear hyperbolic equation

$$
\varepsilon\left(\frac{\partial^{2} u}{\partial t^{2}}-\frac{\partial^{2} u}{\partial x^{2}}\right)-\varphi(t, x, u) \frac{\partial u}{\partial t}+\psi(t, x, u)=0 .
$$

In [25], Trenogin set up asymptotics in a small parameter of the solution of the boundary value problem

$$
\frac{\partial u}{\partial t}-\varepsilon b(x, t) \frac{\partial^{2} u}{\partial x^{2}}+c(x, t, u)=0, u(x, 0)=\varphi(x), u(0, t)=u(l, t)=0 .
$$

In [26], V.Yu. Lunin constructed complete asymptotics of the solution of the Dirichlet problem for the nonlinear elliptic equation

$$
-\varepsilon^{4} \sum_{i=1}^{n} \frac{\partial}{\partial x_{i}}\left(\frac{\partial u}{\partial x_{i}}\right)^{3}-\varepsilon^{2} \sum_{i=1}^{n} \frac{\partial^{2} u}{\partial x_{i}^{2}}+F(x, u)=0
$$

We also note the papers [27]-[32] that were devoted to construction of asymptotics of solutions of different boundary value problems for singularly perturbed nonlinear differential equations. 
The carried out analysis represents the following characteristics of the studied singularly perturbed nonlinear boundary value problems. In the first place, a great majority of singularly perturbed nonlinear equations degenerate for $\varepsilon=0$ either into functional or ordinary differential equations. In the studied equations the derivatives of the sought-for function enter into the equation linearly, and only the sought-for function itself enters non-linearly into the equation. The domains under consideration are bounded and have no viscous adjoint boundaries (under viscous boundary we understand a boundary in the vicinity of which there arises a boundary layer). Finally, all studied singularly perturbed nonlinear partial differential equations relate to one three classical types.

Note some papers [33]-[38] of the first author of this paper, where singularly perturbed quasilinear elliptic and hyperbolic equations are studied in bounded and in unbounded domains, and under degeneration we get partial equations and the derivatives of the sought-for function enter into the equation non-linearly.

In the present paper we consider a boundary value problem for a non-classical type singularly perturbed quasilinear equation of arbitrary odd order degenerating into a hyperbolic equation. The domains where this boundary value problem is studied, has three adjoining viscous boundaries.

In $D=\{(t, x) \mid 0 \leq t \leq 1, \quad 0 \leq x \leq 1\}$ we consider the following boundary value problem

$$
\begin{aligned}
& L_{\varepsilon} u \equiv(-1)^{m} \varepsilon^{2 m} \frac{\partial^{2 m+1} u}{\partial t^{2 m+1}}-\varepsilon^{p} \frac{\partial}{\partial x}\left(\frac{\partial u}{\partial x}\right)^{p}-\varepsilon \frac{\partial^{2} u}{\partial x^{2}}+ \\
&+\frac{\partial u}{\partial t}+\frac{\partial u}{\partial x}+a(t, x) u-f(t, x)=0 \\
&\left.u\right|_{t=0}=\left.\frac{\partial u}{\partial t}\right|_{t=0}=\ldots=\left.\frac{\partial^{m} u}{\partial t^{m}}\right|_{t=0}=0, \quad(0 \leq x \leq 1) \\
&\left.\frac{\partial^{m+1} u}{\partial t^{m+1}}\right|_{t=1}=\left.\frac{\partial^{m+2} u}{\partial t^{m+2}}\right|_{t=1}=\ldots=\left.\frac{\partial^{2 m} u}{\partial t^{2 m}}\right|_{t=1}=0, \quad(0 \leq x \leq 1) \\
&\left.u\right|_{x=0}=0,\left.\quad u\right|_{x=1}=0, \quad(0 \leq t \leq 1)
\end{aligned}
$$

where $p=2 k+1, k$ and $m$ are arbitrary natural numbers, $a(t, x) \geq \gamma^{2}>0$ and $f(t, x)$ are the smooth functions given in $D$.

In this paper, our goal is to construct asymptotic expansion of the solution of boundary value problem (1.6)-(1.9). When constructing asymptotics we are guided by the Vishik-Lusternik technique. It should be noted that it is not succeeded to construct asymptotics of the solution of the problem under consideration by traditional way. In this connection, here the first iterative process and the iterative process that helps to construct boundary layer type functions, are embedded one to another. Furthermore, the notion "satisfaction of boundary condition approximately to within any positive degree of a small parameter" was introduced. In what follows, this notion is used when estimating the residual term. Note that earlier such an approach was used by the first author of this paper in the papers [35], [36].

\section{Carrying Out Iterative Processes}

In the first iterative process, the approximate solution of equation (1.6) is sought in the form

$$
W=W_{0}(t, x)+\varepsilon W_{1}(t, x)+\ldots+\varepsilon^{n} W_{n}(t, x),
$$

and the functions $W_{i}(t, x) ; i=0,1, \ldots, n$ will be chosen so that

$$
L_{\varepsilon} W=0\left(\varepsilon^{n+1}\right) .
$$


Substituting (2.1) in (2.2), expanding the nonlinear term in powers of $\varepsilon$ and grouping the terms with identical powers of $\varepsilon$, for determining $W_{i} ; i=0,1, \ldots, n$ we get the following recurrently connected equations

$$
\begin{aligned}
& \frac{\partial W_{0}}{\partial t}+\frac{\partial W_{0}}{\partial x}+a W_{0}=f(t, x), \\
& \frac{\partial W_{i}}{\partial t}+\frac{\partial W_{i}}{\partial x}+a W_{i}=f_{i}(t, x),
\end{aligned}
$$

where $f_{i}(t, x)$ are the known functions dependent on $W_{0}, W_{1}, \ldots, W_{i-1} ; i=1,2, \ldots, n$ and their derivatives. Here we give formulas only for $f_{1}(t, x): f_{1}(t, x)=\frac{\partial^{2} W_{0}}{\partial x^{2}}$.

For equations (2.3), (2.4) with respect to $x$ we should use the first condition from (1.9), i.e.

$$
\left.W_{i}\right|_{x=0}=0, i=0,1, \ldots, n .
$$

Boundary conditions with respect to $t$ for equations (2.3), (2.4) is given below. Now we note that with respect to $t$ we will use the first condition from (1.8) for $t=0$. Under such a choice of boundary conditions for equations (2.3), (2.4) on the boundary $S_{1}=\{(t, x) \mid t=0, \quad 0 \leq x \leq 1\}, m$ conditions from $m+1$ boundary conditions of (1.7), on the boundary $S_{2}=\{(t, x) \mid t=1, \quad 0 \leq x \leq 1\}$ all $m$ conditions of (1.8), and on the boundary $S_{3}=\{(t, x) \mid 0 \leq t \leq 1, x=1\}$ the second condition of (1.9) will be lost. For compensating the lost boundary conditions we should construct boundary layer functions near the boundaries $S_{1}, S_{2}, S_{3}$.

If as usually for all functions $W_{i}$ entering into expansion $W=\sum_{i=0}^{n} \varepsilon^{i} W_{i}$ we use the condition $\left.W_{i}\right|_{t=0}=0$ and carry out first iterative process and then construct the boundary layer type function $V=\sum_{j=0}^{n_{1}} \varepsilon^{1+j} V_{j}$ near the boundary $S_{1}$, for compensating the lost conditions $\left.\frac{\partial^{k}}{\partial t^{k}}(W+V)\right|_{t=0}=0$; $k=1,2, \ldots, m$, then the sum $W+V$ does not satisfy the boundary condition $\left.(W+V)\right|_{t=0}=0$ which the function $W$ satisfied. To overcome these difficulties, the first iterative process by means of which the functions $W_{i}$ are constructed, and the iterative process that helps to construct the boundary layer type function $V_{j}$ near the boundary $S_{1}$, are embedded one to another. Therefore before finding boundary conditions with respect to $t$, for equations $(2.3),(2.4)$ at first it is necessary to obtain the equations whose solutions will be boundary layer functions near $S_{1}$.

We should look for the boundary layer function $V$ near the boundary $S_{1}$, in the form

$$
V=\varepsilon\left(V_{0}+\varepsilon V_{1}+\ldots+\varepsilon^{n_{1}} V_{n_{1}}\right)
$$

as the solution of the equation

$$
L_{\varepsilon, 1}(W+V)-L_{\varepsilon, 1} W=0\left(\varepsilon^{n_{1}+1}\right),
$$

where $L_{\varepsilon, 1}$ is a new decomposition of the operator $L_{\varepsilon}$ near the boundary $S_{1}$ in the coordinates $(\xi, x)$, where $\xi=\frac{t}{\varepsilon}$. The exact value of $n_{1}$ will be determined later. Now we only note that $n_{1} \geq n+1$.

From (2.6) and (2.7) we get the following recurrently connected equations for determining the functions $V_{j} ; j=0,1, \ldots, n_{1}$ :

$$
\begin{gathered}
(-1)^{m} \frac{\partial^{2 m+1} V_{0}}{\partial \xi^{2 m+1}}+\frac{\partial V_{0}}{\partial \xi}=0, \\
(-1)^{m} \frac{\partial^{2 m+1} V_{j}}{\partial \xi^{2 m+1}}+\frac{\partial V_{j}}{\partial \xi}=h_{j} ; j=1,2, \ldots, n_{1},
\end{gathered}
$$

where $h_{j}$ are the known functions dependent on $V_{0}, V_{1}, \ldots, V_{j-1}$ and their derivatives. We give a formula for $h_{1}(\xi, x)=-\frac{\partial V_{0}}{\partial x}-a(0, x) V_{0}(\xi, x)$. 
The boundary conditions for equations (2.8), (2.9) whose solutions will be the functions $V_{0}, V_{1}, \ldots, V_{n_{1}}$, are obtained from the requirement that the sum $W+V$ for $t=0$ should satisfy conditions of (1.7) except the first condition, i.e.

$$
\left.\frac{\partial}{\partial t}(W+V)\right|_{t=0}=\left.\frac{\partial^{2}}{\partial t^{2}}(W+V)\right|_{t=0}=\ldots=\left.\frac{\partial^{m}}{\partial t^{m}}(W+V)\right|_{t=0}=0 .
$$

Note that (2.10) yields that the number $n_{1}$, in (2.6) should be taken equal to $n+m-1$. In other words, it is necessary to look for the function $V$ in the form

$$
V=\varepsilon\left(V_{0}+\varepsilon V_{1}+\varepsilon^{2} V_{2}+\ldots+\varepsilon^{n+m-1} V_{n+m-1}\right) .
$$

Now we find boundary conditions with respect to $t$ for equations (2.3), (2.4). For that we substitute the expressions $(2.1),(2.11)$ for $W, V$ in the equality

$$
\left.(W+V)\right|_{t=0}=0
$$

and equate to zero the coefficients for $\varepsilon$ whose powers are less than $n+1$. Then we have

$$
\left.W_{0}\right|_{t=0}=0,\left.\quad W_{i}\right|_{t=0}=-\left.V_{i-1}\right|_{\xi=0} ; i=1,2, \ldots, n .
$$

It should be noted that if the functions $W_{i} ; i=0,1, \ldots, n$ satisfy condition $(2.13)$, then for the sum $W+V$ boundary condition $(2.12)$ will be satisfied not exactly but approximately, to within $\varepsilon^{n+1}$, i.e.

$$
\left.(W+V)\right|_{t=0}=\varepsilon^{n+1} \varphi_{\varepsilon}(x) .
$$

Here the function $\varphi_{\varepsilon}(x)$ is determined by the formula

$$
\varphi_{\varepsilon}(x)=\left.\left(V_{n}+\varepsilon V_{n+1}+\ldots+\varepsilon^{m-1} V_{n+m-1}\right)\right|_{\xi=0} .
$$

Having substituted the expressions for $W$ and $V$, respectively from (2.1), (2.11) in (2.10), and comparing the terms at identical powers of $\varepsilon$, we get the following boundary conditions for equations $(2.8),(2.9)$

$$
\begin{aligned}
& \left.\frac{\partial V_{0}}{\partial \xi}\right|_{\xi=0}=-\left.\frac{\partial W_{0}}{\partial t}\right|_{t=0},\left.\frac{\partial^{2} V_{0}}{\partial \xi^{2}}\right|_{\xi=0}=0,\left.\frac{\partial^{3} V_{0}}{\partial \xi^{3}}\right|_{\xi=0}=0, \ldots,\left.\frac{\partial^{m} V_{0}}{\partial \xi^{m}}\right|_{\xi=0}=0 \\
& \left.\frac{\partial V_{1}}{\partial \xi}\right|_{\xi=0}=-\left.\frac{\partial W_{1}}{\partial t}\right|_{t=0},\left.\frac{\partial^{2} V_{1}}{\partial \xi^{2}}\right|_{\xi=0}=\left.\frac{\partial^{2} W_{0}}{\partial t^{2}}\right|_{t=0} \\
& \left.\frac{\partial^{3} V_{1}}{\partial \xi^{3}}\right|_{\xi=0}=0, \ldots,\left.\frac{\partial^{m} V_{1}}{\partial \xi^{m}}\right|_{\xi=0}=0 \\
& \left.\frac{\partial V_{n+m-1}}{\partial \xi}\right|_{\xi=0}=0,\left.\quad \frac{\partial^{2} V_{n+m-1}}{\partial \xi^{2}}\right|_{\xi=0}=0, \ldots,\left.\frac{\partial^{m-1} V_{n+m-1}}{\partial \xi^{m-1}}\right|_{\xi=0}=0, \\
& \left.\frac{\partial^{m} V_{n+m-1}}{\partial \xi^{m}}\right|_{\xi=0}=-\left.\frac{\partial^{m} W_{n}}{\partial t^{m}}\right|_{t=0} .
\end{aligned}
$$

From (2.13) and $\left(2.16_{0}\right)-\left(2.16_{n+m-1}\right)$ it is seen that the functions $W_{i}$ and $V_{j}$ will be constructed 
in turn in the sequence $W_{0}, V_{0}, W_{1}, V_{1}, \ldots, W_{n}, V_{n}, V_{n+1}, \ldots, V_{n+m-1}$. Now construct the functions $W_{0}, W_{1}, W_{n}, \ldots, W_{n}$ and $V_{0}, V_{1}, \ldots, V_{n+m-1}$. From (2.3), (2.5) for $i=0$ and (2.13) we have that $W_{0}$ is the solution of the following boundary value problem

$$
\frac{\partial W_{0}}{\partial t}+\frac{\partial W_{0}}{\partial x}+a(t, x) W_{0}=f(t, x) ;\left.W\right|_{t=0}=0,\left.\quad W_{0}\right|_{x=0}=0 .
$$

Boundary value problem (2.17) is called a degenerated problem corresponding to problem (1.6)(1.9). It should be noted that here the solution of the degenerated problem has breaks on the bisectrix $t=x$ of the first quadrant.

The following statement is valid.

Lemma 2.1. Let $f(t, x) \in C^{2 n+2 m+2}(D), a(t, x) \in C^{2 n+2 m+2}(D)$, the function $f(t, x)$ satisfy the condition

$$
\left.\frac{\partial^{i} f(t, x)}{\partial t^{i_{1}} \partial x^{i_{2}}}\right|_{t=x}=0, i=i_{1}+i_{2} ; i=0,1, \ldots, 2 n+2 m+2 .
$$

Then problem (2.17) has a unique solution, $W_{0}(t, x) \in C^{2 n+2 m+2}(D)$ and

$$
\left.\frac{\partial^{i} W_{0}(t, x)}{\partial t^{i_{1}} \partial x^{i_{2}}}\right|_{t=x}=0, i=i_{1}+i_{2} ; i=0,1, \ldots, 2 n+2 m+2 .
$$

Proof.The characteristical line of the equation in problem (2.17) passing through the origin of coordinates, divides the rectangle $D$ into two parts $D_{1}=\{(t, x) \in D \mid x \leq t\}$ and $D_{2}=\{(t, x) \in D \mid x \geq t\}$. The solution of problem (2.17) may be sought in the form

$$
W_{0}(t, x)= \begin{cases}W_{0}^{(1)}(t, x) & \text { for } \quad(t, x) \in D_{1}, \\ W_{0}^{(2)}(t, x) & \text { for } \quad(t, x) \in D_{2},\end{cases}
$$

the functions $W_{0}^{(1)}$ and $W_{0}^{(2)}$ are the solutions of the following boundary value problem

$$
\begin{gathered}
\frac{\partial W_{0}^{(1)}}{\partial t}+\frac{\partial W_{0}^{(1)}}{\partial x}+a(t, x) W_{0}^{(1)}=f(t, x) ;(t, x) \in D_{1} ; \\
\left.W_{0}^{(1)}\right|_{x=0}=0,(0 \leq t \leq 1), \\
\frac{\partial W_{0}^{(2)}}{\partial t}+\frac{\partial W_{0}^{(2)}}{\partial x}+a(t, x) W_{0}^{(2)}=f(t, x) ;(t, x) \in D_{2} ; \\
\left.W_{0}^{(2)}\right|_{t=0}=0,(0 \leq x \leq 1) .
\end{gathered}
$$

The solutions of problem (2.21), (2.22) are represented by the following formula:

$$
\begin{aligned}
& W_{0}^{(1)}(t, x)=\int_{0}^{x} f(t-x+\tau, \tau) \exp \left[-\int_{\tau}^{x} a(t-x+\xi, \xi) d \xi\right] d \tau, \\
& W_{0}^{(2)}(t, x)=\int_{0}^{t} f(\tau, \tau+x-t) \exp \left[-\int_{\tau}^{t} a(\xi, \xi+x-t) d \xi\right] d \tau .
\end{aligned}
$$

Obviously, if $a(t, x)$ and $f(t, x)$ are rather smooth functions in $D$, then the functions $W_{0}^{(1)}(t, x)$ and $W_{0}^{(2)}(t, x)$ determined by formula (2.23), (2.24) are also smooth functions in $D_{1}$ and $D_{2}$ respectively. Therefore, smoothness of the function $W_{0}(t, x)$ represented by formula (2.20) in $D$ for $x \neq t$ doesn't 
give rise to doubts. But the values of the functions $W_{0}^{(1)}(t, x)$ and $W_{0}^{(2)}(t, x)$ do not coincide on the line $x=t$. Consequently, the function $W_{0}(t, x)$ have breaks for $x=t$.

Using the explicit form (2.23), (2.24) of functions $W_{0}^{(1)}(t, x)$ and $W_{0}^{(2)}(t, x)$ it is easy to see that when $f(t, x)$ satisfies condition (2.18), all derivatives $W_{0}^{(1)}(t, x)$ and $W_{0}^{(2)}(t, x)$ to the $(2 n+2 m+2)$ th order inclusively vanish for $t=x$. Therefore, from (2.20) it follows that

$$
\begin{gathered}
\left.\frac{\partial^{i} W_{0}(t, x)}{\partial t^{i_{1}} \partial x^{i_{2}}}\right|_{x-t=-0}=\left.\frac{\partial^{i} W_{0}^{(1)}(t, x)}{\partial t^{i_{1}} \partial x^{i_{2}}}\right|_{t=x}= \\
=\left.\frac{\partial^{i} W_{0}^{(2)}(t, x)}{\partial t^{i_{1}} \partial x^{i_{2}}}\right|_{t=x}=\left.\frac{\partial^{i} W_{0}(t, x)}{\partial t^{i_{1}} \partial x^{i_{2}}}\right|_{x-t=+0}=0 .
\end{gathered}
$$

Consequently, the functions $\frac{\partial^{i} W_{0}(t, x)}{\partial t^{i} \partial x^{i}{ }^{2}} ; i=1,2, \ldots, 2 n+2 m+2$ are continuous and vanish for $t=x$, i.e. condition (2.19) is fulfilled. Lemma 2.1 is proved.

From (2.13) for $i=0$ it follows that before constructing the function $W_{1}$ it is necessary to determine $V_{0}$. The function $V_{0}$ is the boundary layer type solution of equation (2.8) satisfying boundary conditions $\left(2.16_{0}\right)$. The characteristical equation corresponding to ordinary differential equation (2.8) has $m$ roots with negative real parts. Denote them by $\lambda_{1}, \lambda_{2}, \ldots, \lambda_{m}$. It should be noted that the amount of lost boundary conditions on $S_{1}$ also equals $m$. Therefore, problem (1.6)-(1.9) regularly degenerates on $S_{1}$. It is easy to show that the boundary layer type solution of problem $(2.8),\left(2.16_{0}\right)$ is of the form

$$
\begin{gathered}
V_{0}(\xi, x)= \\
=-\frac{\partial W_{0}(0, x)}{\partial t}\left[C_{01} \exp \left(\lambda_{1} \xi\right)+C_{02} \exp \left(\lambda_{2} \xi\right)+\ldots+C_{0 m} \exp \left(\lambda_{m} \xi\right)\right],
\end{gathered}
$$

where $C_{0 i}$ are the known numbers.

As the functions $W_{0}, V_{0}$ are known, then we can already determine the function $W_{1}$ from problem (2.4), (2.5), (2.13) for $i=1$. The solution of this problem may be sought in the form $W_{1}=$ $W_{1}^{(1)}+W_{1}^{(2)}$, where $W_{1}^{(1)}$ and $W_{1}^{(2)}$ are the solutions of the following boundary value problems:

$$
\begin{aligned}
& \frac{\partial W_{1}^{(1)}}{\partial t}+\frac{\partial W_{1}^{(2)}}{\partial t}+a(t, x) W_{1}^{(1)}=\frac{\partial^{2} W_{0}}{\partial x^{2}} ;\left.W_{1}^{(1)}\right|_{t=0}=0,\left.\quad W_{1}^{(1)}\right|_{x=0}=0 \\
& \frac{\partial W_{1}^{(2)}}{\partial t}+\frac{\partial W_{1}^{(2)}}{\partial t}+a(t, x) W_{1}^{(2)}=0,\left.\quad W_{1}^{(2)}\right|_{t=0}=\varphi_{1}(x),\left.\quad W_{1}^{(2)}\right|_{x=0}=0 .
\end{aligned}
$$

Here $\varphi_{1}(x)$ is determined by the formula

$$
\varphi_{1}(x)=-V_{0}(0, x)=-\left(\sum_{i=1}^{m} C_{0 i}\right) \frac{\partial W_{0}(0, x)}{\partial t} .
$$

The first part of the equation for $W_{1}^{(1)}$ satisfies condition (2.18) in lemma 2.1 for $i=0,1, \ldots, 2 n+2 m$. Therefore, by the same lemma, problem (2.26) has a unique solution, $W_{1}^{1}(t, x) \in C^{2 n+2 m}(D)$ and the function $W_{1}^{(1)}(t, x)$ satisfies the condition

$$
\left.\frac{\partial^{i} W_{1}^{(1)}(t, x)}{\partial t^{i_{1}} \partial x^{i_{2}}}\right|_{t=x}=0 ; i=i_{1}+i_{2} ; i=0,1, \ldots, 2 n+2 m .
$$


The solution of problem (2.27) is represented by the formula

$$
W_{1}^{(2)}(t, x)=\left\{\begin{array}{l}
0 \\
\varphi_{1}(t-x) \exp \left[-\int_{0}^{t} a(\tau, x-t+\tau) d \tau\right] \quad \text { for } 0 \leq t<x \leq 1 .
\end{array}\right.
$$

By lemma 2.1, from (2.28) it follows that $\varphi_{1}(x) \in C^{2 n+2 m+1}[0,1]$. Therefore, smoothness in $D$ for $t \neq x$, of the function $W_{1}^{(2)}(t, x)$ determined by formula (2.30) is obvious. From (2.19) and (2.28) we get

$$
\varphi_{1}^{(k)}(0)=0 ; \quad k=0,1, \ldots, 2 n+2 m+1 .
$$

Taking into account (2.31), the smoothness of the function $W_{1}^{(2)}(t, x)$ and vanishing of all derivatives for $t=x$ is obtained directly from (2.30). Hence and from (2.29) it follows that the function $W_{1}(t, x)$ being the sum of $W_{1}^{(1)}, W_{1}^{(2)}$ belongs to the space $C^{2 n+2 m}(D)$ and satisfies the condition

$$
\left.\frac{\partial^{i} W_{1}(t, x)}{\partial t^{i_{1}} \partial x^{i_{2}}}\right|_{t=0}=0 ; \quad i=i_{1}+i_{2} ; i=0,1, \ldots, 2 n+2 m .
$$

The remaining functions $W_{2}, W_{3}, \ldots, W_{n}$ entering into the right hand side of (2.1) are constructed by the reasonings similar to ones carried out for $W_{1}$. Therefore we will not stop on their construction. By constructing the functions $V_{0}, V_{1}, \ldots, V_{n+m-1}$ the following statement is used.

Lemma 2.2. The functions $V_{j}$ being the boundary layer type solutions of equation (2.9), satisfying appropriate boundary conditions from $\left(2.16_{1}\right)-\left(2.16_{n+m-1}\right)$ are determined by the formula

$$
\begin{gathered}
V_{j}(\xi, x)=\sum_{i=1}^{m}\left[C_{j 0}^{(i)}(x)+C_{j 1}^{(i)}(x) \xi+\ldots+C_{j j}^{(i)}(x) \xi^{j}\right] \exp (\lambda, \xi), \\
j=1,2, \ldots, n+m-1,
\end{gathered}
$$

and the coefficients $C_{j s}^{(i)}(x)$ are uniformly expressed by the function

$$
\frac{\partial^{k} W_{r}(0, x)}{\partial t^{k_{1}+1} \partial x^{k_{2}}} ; k=k_{1}+k_{2}+1 ; r=0,1, \ldots, j ; \quad k_{1}=0,1, \ldots, m-1 ; k_{1}+k_{2}+r=j .
$$

Proof. At first determine the function $V_{1}$. The function $V_{1}$ is the boundary layer type solution of equation (2.9) for $j=1$, satisfying boundary condition $\left(2.16_{1}\right)$. Taking into account explicit form (2.25) of the function $V_{0}(\xi, x)$, we represent the right hand side of equation (2.9) for $j=1$ in the form

$$
h_{1}(\xi, x)=\theta(x)\left[\sum_{i=1}^{m} C_{0 i} \exp \left(\lambda_{i} \xi\right)\right]
$$

and $\theta(x)$ is determined by the formula

$$
\theta(x)=\frac{\partial^{2} W_{0}(0, x)}{\partial t \partial x}+a(0, x) \frac{\partial W_{0}(0, x)}{\partial t} .
$$

Obviously, the function

$$
V_{1}^{(1)}(\xi, x)=\theta(x) \xi\left[\sum_{i=1}^{m} b_{0 i} \exp \left(\lambda_{i} \xi\right)\right]
$$


is a boundary layer type particular solution of equation (2.9), where $b_{0 i}$ are known numbers. Represent $V_{1}$ in the form $V_{1}=V_{1}^{(1)}+V_{1}^{(2)}$. Then $V_{1}^{(2)}$ will be a boundary layer type solution of the following boundary value problem

$$
\begin{gathered}
(-1)^{m} \frac{\partial^{2 m+1} V_{1}^{(2)}}{\partial \xi^{2 m+1}}+\frac{\partial V_{1}^{(2)}}{\partial \xi}=0 \\
\left.\frac{\partial V_{1}}{\partial \xi}\right|_{\xi=0}=d_{1}(x),\left.\frac{\partial^{2} V_{1}^{(2)}}{\partial \xi^{2}}\right|_{\xi=0}=d_{2}(x), \ldots,\left.\frac{\partial^{m} V_{1}^{(2)}}{\partial \xi^{m}}\right|_{\xi=0}=d_{m}(x) .
\end{gathered}
$$

Here the following denotation are used:

$$
\begin{gathered}
d_{1}(x)=-\left.\frac{\partial W_{1}}{\partial t}\right|_{t=0}+p_{1} \theta(x), \quad d_{2}(x)=-\left.\frac{\partial^{2} W_{1}}{\partial t^{2}}\right|_{t=0}+p_{2} \theta(x), \\
d_{j}(x)=p_{j} \theta(x) ; j=3,4, \ldots, m \\
p_{s}=-s \sum_{i=1}^{m} b_{0 i} \lambda_{i}^{s-1} ; s=1,2, \ldots, m .
\end{gathered}
$$

It is easy to see that the boundary layer type solution of problem $(2.38),(2.39)$ is of the form:

$$
V_{1}^{(2)}(\xi, x)=C_{1}(x) \exp \left(\lambda_{1} \xi\right)+C_{2}(x) \exp \left(\lambda_{2} \xi\right)+\ldots+C_{m}(x) \exp \left(\lambda_{m} \xi\right)
$$

Here the functions $C_{i}(x)$ are represented by the following formula:

$$
\begin{gathered}
C_{i}(x)=C_{i}^{(1)} \frac{\partial W_{1}(0, x)}{\partial t}+C_{i}^{(2)} \frac{\partial^{2} W_{0}(0, x)}{\partial t^{2}}+C_{i}^{(3)} \frac{\partial^{2} W_{0}(0, x)}{\partial t \partial x}+ \\
+C_{i}^{(4)} a(0, x) \frac{\partial W_{0}(0, x)}{\partial t},
\end{gathered}
$$

where $C_{i}^{(1)}, C_{i}^{(2)}, C_{i}^{(3)}, C_{i}^{(4)} ; i=1,2, \ldots, m$ are known numbers.

From (2.37) and (2.40) we get that the function $V_{1}$ being the sum of $V_{1}^{(1)}$ and $V_{1}^{(2)}$ is determined by the formula:

$$
V_{1}(\xi, x)=\sum_{i=1}^{n}\left[C_{i}(x)+b_{0 i} \theta(x) \xi\right] \exp \left(\lambda_{i} \xi\right) .
$$

Introducing the denotation

$$
C_{10}^{(i)}(x)=C_{i}(x), C_{11}^{(i)}(x)=b_{0 i} \theta(x) ; i=1,2, \ldots, m,
$$

we can write formula $(2.42)$ as

$$
V_{1}(\xi, x)=\sum_{i=1}^{m}\left[C_{10}^{(i)}(x)+C_{11}^{(i)}(x) \xi\right] \exp \left(\lambda_{i} \xi\right) .
$$

From (2.36), (2.41), (2.43), (2.44) it follows that the statement of lemma 2.2 is valid for $j=1$.

Assuming that the statement of lemma 2.2 is valid for $j \leq r-1, \quad(r<n+m-1)$, and repeating the reasonings carried out when determining the function $V_{1}$, we can prove that it is valid for $j=r$ as well. 
Lemma 2.2 is proved.

Multiply all $V_{j}$, by smoothing functions and denote the obtained new functions again by $V_{j} ; j=$ $0,1, \ldots, n+m+1$.

It is known that the functions $W_{i}(t, x) ; i=0,1, \ldots, n$ together will all their derivatives vanish for $x=t$ and in particular for $x=t=0$. Consequently, from (2.25), (2.33), (2.34) we get that all functions $V_{j}(\xi, x) ; j=0,1, \ldots, n+m-1$ vanish for $x=0$. Hence and from (2.1), (2.5), (2.11) it follows that the sum $W+V$ in addition to (2.10), (2.14) satisfies the boundary condition

$$
\left.(W+V)\right|_{x=0}=0
$$

as well. The constructed sum $W+V$ generally speaking, doesn't satisfy boundary conditions (1.9) on $S_{2}$. In this connection, it is necessary to construct a boundary layer type function near the boundary $S_{2}$.

Construction of boundary layer functions near the boundary $S_{2}$ is performed similar to construction of boundary layer functions near the boundary $S_{1}$. Therefore, we will not stop in detail on construction of boundary layer functions near the boundary $S_{2}$. We note only some moments.

Here, change of variables is made by the formulas: $1-t=\varepsilon y, x=x$. The boundary layer type function $\eta$ near the boundary $S_{2}$ should be sought in the form:

$$
\eta=\varepsilon^{m+1}\left(\eta_{0}+\varepsilon \eta_{1}+\ldots+\varepsilon^{n+m-1} \eta_{n+m-1}\right),
$$

as the solution of the equation

$$
L_{\varepsilon, 2}(W+V+\eta)-L_{\varepsilon, 2}(W+V)=O\left(\varepsilon^{n+2 m+1}\right),
$$

where $L_{\varepsilon, 2}$ is a new decomposition of the operator $L_{\varepsilon}$ near the boundary $S_{2}$ in coordinates $(y, x)$.

The equations for $\eta_{0}, \eta_{1}, \ldots, \eta_{n+m-1}$ have the same form as the equations for $V_{0}, V_{1}, \ldots, V_{n+m-1}$. The right hand sides of equations for $\eta_{1}, \eta_{2}, \ldots, \eta_{n+m-1}$ differ from the right hand sides of appropriate equations for $V_{1}, V_{2}, \ldots, V_{n+m-1}$ only by opposite signs.

The boundary conditions for equations whose solutions will be the functions $\eta_{0}, \eta_{1}, \ldots, \eta_{n+m-1}$ are found from the requirement that the sum $W+V+\eta$ should satisfy the following boundary conditions:

$$
\begin{gathered}
\left.\frac{\partial^{m+1}}{\partial t^{m+1}}(W+V+\eta)\right|_{t=1}= \\
=\left.\frac{\partial^{m+2}}{\partial t^{m+2}}(W+V+\eta)\right|_{t=1}=\ldots=\left.\frac{\partial^{2 m}}{\partial t^{2 m}}(W+V+\eta)\right|_{t=1}=0 .
\end{gathered}
$$

The following statement is proved similar to the proof of lemma 2.2.

Lemma 2.3. The boundary layer type functions near the boundary $S_{2}$ are determined by the formula

$$
\begin{gathered}
\eta_{j}(y, x)=\sum_{i=1}^{m}\left[d_{j 0}^{(i)}(x)+d_{j 1}^{(i)}(x) y+\ldots+d_{j j}^{(i)}(x) y^{j}\right] \exp \left(\lambda_{i} y\right) \\
j=0,1, \ldots, n+m-1 .
\end{gathered}
$$

Here the coefficients $d_{j s}^{(i)}(x)$ are expressed by the function

$$
\begin{gathered}
\frac{\partial^{m+1+k} W_{r}(1, x)}{\partial t^{m+1+k_{1}} \partial x^{k_{2}}} \\
k=k_{1}+k_{2} ; k_{1}=0,1, \ldots, m-1 ; r=0,1, \ldots, j ; k_{1}+k_{2}+r=j .
\end{gathered}
$$


Multiply all functions $\eta_{j}$ by the smoothing function and for the obtained new functions leave the previous denotation $\eta_{j} ; j=0,1, \ldots, n+m-1$.

As the function $\eta$ vanishes at the expense of the smoothing function for $t=0$, then from (2.10) and (2.14) if follows that the sum $W+V+\eta$ along with conditions (2.48) satisfies the following boundary conditions as well:

$$
\begin{gathered}
\left.(W+V+\eta)\right|_{t=0}=\varepsilon^{n+1} \varphi_{\varepsilon}(x),\left.\frac{\partial}{\partial t}(W+V+\eta)\right|_{t=0}= \\
=\left.\frac{\partial^{2}}{\partial t^{2}}(W+V+\eta)\right|_{t=0}=0, \ldots,\left.\frac{\partial^{m}}{\partial t^{m}}(W+V+\eta)\right|_{t=0}=0
\end{gathered}
$$

where $\varphi_{\varepsilon}(x)$ is determined by formula $(2.15)$.

Following (2.45) and (2.46), we have that if all functions $\eta_{j}$ vanish for $x=0$, i.e.

$$
\left.\eta_{j}\right|_{x=0}=0 ; j=0,1, \ldots, n+m-1,
$$

then the sum $W+V+\eta$ in addition to (2.48), (2.51) will satisfy the boundary condition

$$
\left.(W+V+\eta)\right|_{x=0}=0,
$$

as well.

If follows from (2.49), (2.50) that for fulfilment of conditions (2.52) it is suffices that the functions $W_{i}$ satisfy the conditions

$$
\begin{gathered}
\frac{\partial^{m+1+k} W_{i}(1,0)}{\partial t^{m+1+k_{1}} \partial x^{k_{2}}}=0 \\
k=k_{1}+k_{2} ; i=0,1, \ldots, n ; k_{1}+k_{2}+r=0,1, \ldots, n+m-1 .
\end{gathered}
$$

Assume that the function $f(t, x)$ satisfies the condition

$$
\frac{\partial^{k} f(1,0)}{\partial t^{k_{1}} \partial x^{k_{2}}}=0 ; k=k_{1}+k_{2} ; k=0,1, \ldots, n+m-1 .
$$

Then conditions (2.54) and consequently (2.52), (2.53) will be satisfied.

Thus, the constructed sum $W+V+\eta$ satisfies boundary conditions (2.48), (2.51), (2.53). But this sum do not satisfy boundary conditions of the second boundary condition from (1.9) for $x=1$. Therefore we should construct a boundary layer type function $\psi$ near the boundary $S_{3}$ so that $\psi$ could ensure fulfilment of the boundary condition

$$
\left.(W+V+\eta+\psi)\right|_{x=1}=0
$$

By constructing the function $\psi$ we should care that the equality

$$
L_{\varepsilon, 3}(W+V+\eta+\psi)-L_{\varepsilon, 3}(W+V+\eta)=O\left(\varepsilon^{n+1}\right)
$$

be fulfilled. Here $L_{\varepsilon, 3}$ denotes a new decomposition of the operator $L_{\varepsilon}$ near the boundary $S_{3}$ in coordinates $(t, \tau)$, where $\tau=\frac{1-x}{\varepsilon}$. We should look for the boundary layer function $\psi$ in the form

$$
\psi=\psi_{0}(t, \tau)+\varepsilon \psi_{1}(t, \tau)+\ldots+\varepsilon^{n+1} \psi_{n+1}(t, \tau) .
$$


Having substituted expression (2.58) of the function $\psi$ and new expansions in a small parameter of already constructed functions $W, V, \eta$ in coordinates $(t, \tau)$ in $(2.57)$ we get the following equations whose solutions are the functions $\psi_{0}, \psi_{1}, \ldots, \psi_{n+1}$ :

$$
\begin{gathered}
\frac{\partial}{\partial \tau}\left(\frac{\partial \psi_{0}}{\partial \tau}\right)^{p}+\frac{\partial^{2} \psi_{0}}{\partial \tau^{2}}+\frac{\partial \psi_{0}}{\partial \tau}=0 \\
\frac{\partial}{\partial \tau}\left\{\left[p\left(\frac{\partial \psi_{0}}{\partial \tau}\right)^{p-1}+1\right] \frac{\partial \psi_{j}}{\partial \tau}\right\}+\frac{\partial \psi_{j}}{\partial \tau}=\Phi_{j} ; j=1,2, \ldots, n+1
\end{gathered}
$$

Here $\Phi_{j}$ are known functions polynomially depending on the first and second derivatives of the functions $V_{0}, V_{1}, \ldots, V_{j-1}$.

We now find boundary conditions for equations (2.59), (2.60). As is known, all functions $W_{i}(t, x)$; $i=0,1, \ldots, n$ vanish for $t=x$, in particular for $t=x=1$. Taking this fact into account, from (2.49) and (2.50) we get that all functions $\eta_{j} ; j=0,1, \ldots, n+m-1$ vanish for $x=1$, whence we have $\left.\eta\right|_{x=1}=0$. Hence it follows that we can write equality (2.56) in the form

$$
\left.(W+V+\psi)\right|_{x=1}=0
$$

Assume that the function $f(t, x)$ satisfies the condition

$$
\frac{\partial^{k} f(0,1)}{\partial t^{k_{1}} \partial x^{k_{2}}}=0 ; \quad k=k_{1}+k_{2} ; \quad k=0,1, \ldots, n+m-1 .
$$

Then from $(2.33),(2.34)$ it follows that all functions $V_{j} ; j=0,1, \ldots, n+m-1$ will vanish for $x=1$, hence we have $\left.V\right|_{x=1}=0$. Therefore, equality (2.61) takes the form

$$
\left.(W+\psi)\right|_{x=1}=0 .
$$

Having substituted expressions (2.1), (2.58) for $W, \psi$ in (2.63), and taking into account the fact that all functions $\psi_{j} ; j=0,1, \ldots, n+1$, should be boundary layer type functions from (2.63) we get the following conditions for equations (2.59), (2.60):

$$
\left.\psi_{j}\right|_{\tau=0}=\varphi_{j}(t), \quad \lim _{\tau \rightarrow+\infty} \psi_{j}=0
$$

where $\varphi_{j}(t)=-W_{j}(t, 1)$, for $j=0,1, \ldots, n$ and $\varphi_{n+1} \equiv 0$.

The following statement is valid.

Lemma 2.4. For every $t \in[0,1]$ problem (2.59), (2.64) for $j=0$ has a unique solution, and the function $\psi_{0}(t, \tau)$ to $\tau$ with respect to $\tau$ is continuously differentiable with respect to $t$, has continuous derivatives to $2(n+m+1)$-th order, inclusively. The following estimation is valid:

$$
\left|\frac{\partial^{i} \psi_{0}(t, \tau)}{\partial t^{i_{1}} \partial \tau^{i_{2}}}\right| \leq G_{i_{1} i_{2}}\left(\left|\varphi_{0}(t)\right|,\left|\varphi_{0}^{\prime}(t)\right|, \ldots,\left|\varphi_{0}^{\left(i_{1}\right)}(t)\right|\right) \exp (-\tau)
$$

where $i=i_{1}+i_{2} ; i=0,1, \ldots, 2 n+m+2 ; G_{i_{1} i_{2}}\left(t_{1}, t_{2}, \ldots, t_{i_{1}+1}\right)$ are some known polynomials of their own arguments with non-negative coefficients, free terms of these polynomials equal zero, and at least one of other coefficients is non zero.

The proof of lemma 2.4 is in [34, theorem 2], (see also [39, theorem 2]).

Construction of remaining functions $\psi_{1}, \psi_{2}, \ldots, \psi_{n+1}$ as the solutions of linear problems (2.60), (2.64) for $j=1,2, \ldots, n+1$ is based on the following statement, whose prove is given in [34, Theorem 3]. 
Lemma 2.5. Problems (2.60), (2.64) for $j=1,2, \ldots, n+1$ have unique solutions, the functions $\psi_{j}(t, \tau) ; j=1,2, \ldots, n+1$ have continuous derivatives to the $2(n+m+1-j)$-th order, inclusively. The following estimations are valid:

$$
\left|\frac{\partial^{i} \psi_{j}(t, \tau)}{\partial t^{i_{1}} \partial \tau^{i_{2}}}\right| \leq \sum_{s=0}^{i_{1}+j+1}\left|a_{j s}(t)\right| \tau^{s} \exp (-\tau),
$$

where $i=i_{1}+i_{2} ; i=0,1, \ldots, 2 n+2 m+2-j ; a_{j s}(t)$ are known functions expressed by the functions $\varphi_{0}(t), \varphi_{1}(t), \ldots, \varphi_{j}(t)$ and their derivatives in the form of polynomials without free terms $j=1,2, \ldots, n+1$.

Multiply all functions $\psi_{j} ; j=0,1, \ldots, n+1$ by the smoothing cofactor and for the obtained new functions leave the previous denotation.

So, we constructed the sum

$$
\tilde{u}=\sum_{i=0}^{n} \varepsilon^{i} W_{i}+\sum_{s=0}^{n+m-1} \varepsilon^{1+s} V_{s}+\sum_{s=0}^{n+m-1} \varepsilon^{1+m+s} \eta_{s}+\sum_{j=0}^{n+1} \varepsilon^{i} \psi_{j},
$$

that following (2.56) satisfies the boundary condition

$$
\left.\tilde{u}\right|_{x=1}=0 \text {. }
$$

As the function $\psi$ vanishes for $x=0$ at the expense of the smoothing cofactor, it follows from (2.53) that in addition to condition (2.68), $\tilde{u}$ satisfies the boundary condition

$$
\left.\tilde{u}\right|_{x=0}=0
$$

as well.

From (2.62) it follows that the functions $\varphi_{j}(t)=-W_{j}(t, 1) ; j=0,1, \ldots, n$ satisfy the following conditions

$$
\varphi_{j}^{(k)}(0)=0 ; k=0,1, \ldots, m ; j=0,1, \ldots, n .
$$

Then from estimations (2.65), (2.66) and from (2.70) we have

$$
\left.\frac{\partial^{k} \psi_{j}(t, \tau)}{\partial t^{k}}\right|_{t=0}=0 ; \quad k=0,1, \ldots, m ; j=0,1, \ldots, n+1 .
$$

From (2.58) and (2.71) we get

$$
\left.\psi\right|_{t=0}=\left.\frac{\partial \psi}{\partial t}\right|_{t=0}=\ldots=\left.\frac{\partial^{m} \psi}{\partial t^{m}}\right|_{t=0}=0
$$

Taking into account $(2.51),(2.72)$ we have that the sum $\tilde{u}=W+V+\eta+\varphi$ alongside with conditions (2.68), (2.69) satisfies the following boundary conditions as well

$$
\left.\tilde{u}\right|_{t=0}=\varepsilon^{n+1} \varphi_{\varepsilon}(x),\left.\quad \frac{\partial \tilde{u}}{\partial t}\right|_{t=0}=\left.\frac{\partial^{2} \tilde{u}}{\partial t^{2}}\right|_{t=0}=\ldots=\left.\frac{\partial^{m} \tilde{u}}{\partial t^{m}}\right|_{t=0}=0 .
$$

It is known that all functions $W_{i}(t, x) ; i=0,1, \ldots, n$ together with their own derivatives vanish at $t=x$, in particular for $t=x=1$. Hence it follows that the function $\varphi_{j}(t)=-W_{j}(t, 1)$ satisfies the conditions:

$$
\varphi_{j}^{(k)}(1)=0 ; k=0,1, \ldots, 2 m .
$$


From estimations (2.65), (2.66) and (2.74) we get that

$$
\left.\frac{\partial^{m+k} \psi_{j}(t, \tau)}{\partial t^{m+1}}\right|_{i=1}=0 ; \quad k=1,2, \ldots, m ; j=0,1, \ldots, n+1 .
$$

From (2.58) and (2.75) it follows that

$$
\left.\frac{\partial^{m+1} \psi}{\partial t^{m+1}}\right|_{t=1}=\left.\frac{\partial^{m+2} \psi}{\partial t^{m+2}}\right|_{t=1}=\ldots=\left.\frac{\partial^{2 m} \psi}{\partial t^{2 m}}\right|_{t=1}=0
$$

Following (2.48), (2.76) we get that the function $\tilde{u}$ in addition to conditions (2.68), (2.69), (2.73), satisfies the following boundary conditions as well:

$$
\left.\frac{\partial^{m+1} \tilde{u}}{\partial t^{m+1}}\right|_{t=1}=\left.\frac{\partial^{m+2} \tilde{u}}{\partial t^{m+2}}\right|_{t=1}=\ldots=\left.\frac{\partial^{2 m} \tilde{u}}{\partial t^{2 m}}\right|_{t=1}=0 .
$$

Introduce the denotation

$$
u-\tilde{u}=z
$$

and call the function $z$ a remainder term, where $u$ is the solution of problem (1.6)-(1.9). Then (2.67), (2.78) yields the following asymptotic expansion in a small parameter of the solution of problem (1.6)-(1.9):

$$
u=\sum_{i=0}^{n} \varepsilon^{i} W_{i}+\sum_{s=0}^{n+m-1} \varepsilon^{1+s} V_{s}+\sum_{s=0}^{n+m-1} \varepsilon^{1+m+s} \eta_{s}+\sum_{j=0}^{n+1} \varepsilon^{j} \psi_{j}+z .
$$

Now we should estimate the remainder term.

\section{Estimating the Remainder Term, and Formulation of the Main Result}

The following lemma is valid.

Lemma 3.1. For the remainder term $z$ in (2.79) the following estimation is valid

$$
\begin{gathered}
\varepsilon^{2 m} \int_{0}^{1}\left(\left.\frac{\partial^{m} z}{\partial t^{m}}\right|_{t=1}\right)^{2} d x+\varepsilon^{p} \iint_{D}\left(\frac{\partial z}{\partial x}\right)^{p+1} d t d x+\varepsilon \iint_{D}\left(\frac{\partial z}{\partial x}\right)^{2} d t d x+ \\
+C_{1} \iint_{D} z^{2} d t d x \leq C_{2} \varepsilon^{2(n+1)}
\end{gathered}
$$

where $C_{1}>0, C_{2}>0$ are constants independent of $\varepsilon$.

Proof. Putting together (2.2), (2.7), (2.47), (2.57), we have that the function $\tilde{u}$ satisfies the equation

$$
L_{\varepsilon} \tilde{u}=O\left(\varepsilon^{n+1}\right) \text {. }
$$


Subtracting (3.2) from (1.6), we get

$$
\begin{gathered}
(-1)^{m} \varepsilon^{2 m} \frac{\partial^{2 m+1} z}{\partial t^{2 m+1}}-\varepsilon^{p} \frac{\partial}{\partial x}\left[\left(\frac{\partial u}{\partial x}\right)^{p}-\left(\frac{\partial \tilde{u}}{\partial x}\right)^{p}\right]-\varepsilon^{2} \frac{\partial^{2} z}{\partial x^{2}}+ \\
+\frac{\partial z}{\partial t}+a z=O\left(\varepsilon^{n+1}\right)
\end{gathered}
$$

From (1.7)-(1.9), (2.68), (2.69), (2.73), (2.77) it follows that $z$ satisfies the following boundary conditions

$$
\begin{gathered}
\left.z\right|_{t=0}=-\varepsilon^{n+1} \varphi_{\varepsilon}(x),\left.\quad \frac{\partial^{k} z}{\partial t^{k}}\right|_{t=0}=0 ;\left.\quad \frac{\partial^{m+k} z}{\partial t^{m+k}}\right|_{t=1}=0 ; \quad k=1,2, \ldots, m \\
\left.z\right|_{x=0}=\left.z\right|_{x=1}=0
\end{gathered}
$$

Here the function $\varphi_{\varepsilon}(x)$ is determined by formula (2.15) and satisfies the conditions

$$
\varphi_{\varepsilon}(0)=\varphi_{\varepsilon}(1)=0
$$

Let us consider the auxiliary function

$$
z_{1}=\varepsilon^{n+1}\left[t^{m+1}(1-t)^{2 m+1} x(1-x)-\varphi_{\varepsilon}(x)\right] .
$$

It is easy to see that the function $z_{1}$ determined by formula (3.7), satisfies boundary conditions (3.4), (3.5) as well.

Represent the remainder term $z$ in the form

$$
z=z_{1}+z_{2}
$$

Obviously, the function $z_{2}$ satisfies the following boundary conditions

$$
\begin{gathered}
\left.\frac{\partial^{k} z_{2}}{\partial t^{k}}\right|_{t=0}=0 ; k=0,1, \ldots, m ;\left.\quad \frac{\partial^{m+k} z_{2}}{\partial t^{m+k}}\right|_{t=0}=0 ; \quad k=1,2, \ldots, m \\
\left.z_{2}\right|_{x=0}=\left.z_{2}\right|_{x=1}=0 .
\end{gathered}
$$

Having substituted the expression of $z$ from (3.8) in (3.3) and taking into account (3.7), after some transformations we get the equation

$$
\begin{gathered}
(-1)^{m} \varepsilon^{2 m} \frac{\partial^{2 m+1} z_{2}}{\partial t^{2 m+1}}-\varepsilon^{p} \frac{\partial}{\partial x}\left\{\left[\frac{\partial\left(z_{2}+\tilde{u}+z_{1}\right)}{\partial x}\right]^{p}-\left[\frac{\partial\left(\tilde{u}+z_{1}\right)}{\partial x}\right]^{p}\right\}- \\
-\varepsilon^{p} \frac{\partial}{\partial x}\left\{\left[\frac{\partial\left(\tilde{u}+z_{1}\right)}{\partial x}\right]^{p}-\left(\frac{\partial \tilde{u}}{\partial x}\right)^{p}\right\}-\varepsilon \frac{\partial^{2} z_{2}}{\partial x^{2}}+\frac{\partial z_{2}}{\partial x}+\frac{\partial z_{2}}{\partial t}+a z_{2}=O\left(\varepsilon^{n+1}\right) .
\end{gathered}
$$

Taking into account (3.7), we can show that the term $\frac{\partial}{\partial x}\left\{\left[\frac{\partial\left(\tilde{u}+z_{1}\right)}{\partial x}\right]^{p}-\left(\frac{\partial \tilde{u}}{\partial x}\right)^{p}\right\}$ has order of 
smallness $O\left(\varepsilon^{n+1}\right)$ with respect to $\varepsilon$. Taking this term to the right hand side of (3.11) we get the following equation

$$
\begin{gathered}
(-1)^{m} \varepsilon^{2 m} \frac{\partial^{2 m+1} z_{2}}{\partial t^{2 m+1}}-\varepsilon^{p} \frac{\partial}{\partial x}\left\{\left[\frac{\partial\left(z_{2}+\tilde{u}+z_{1}\right)}{\partial x}\right]^{p}-\left[\frac{\partial\left(\tilde{u}+z_{1}\right)}{\partial x}\right]^{p}\right\}- \\
-\varepsilon \frac{\partial^{2} z_{2}}{\partial x^{2}}+\frac{\partial z_{2}}{\partial x}+\frac{\partial z_{2}}{\partial t}+a z_{2}=O\left(\varepsilon^{n+1}\right) .
\end{gathered}
$$

Multiplying both hand sides of (3.12) by $z_{2}$, integrating by parts the obtained expressions on domain $D$, allowing for conditions (3.9), (3.10) after certain transformations we get the validity of estimation (3.1) for the function $z_{2}$. From (3.7), (3.8) and from the estimation for $z_{2}$ we get validity of estimation (3.1) for $z$.

Lemma 3.1 is proved.

\section{Conclusion}

Summarizing the obtained results, we arrive at the following statement.

Theorem 4.1. Assume that the function $f(t, x) \in C^{2 n+2 m+2}(D)$ and satisfies conditions (2.18), (2.55), (2.62). Then for the solution of boundary value problem (1.6)-(1.9) the asymptotic representation (2.79) is valid, where the functions $W_{j}$ are determined by the first iterative process, $V_{s}, \eta_{s}, \psi_{j}$ are boundary layer type functions near the boundaries $t=0, t=1$ and $x=1$ and also are determined by appropriate iterative processes, $z$ is a remainder term and estimation (3.1) is valid for it.

\section{Competing Interests}

Authors have declared that no competing interests exist.

\section{References}

[1] Friedrichs KO. Asymptotic phenomenon in mathematical physics. Mathematics Periodic collection of translations of papers in foreign languages. 1957; 1(2):79-84.

[2] Trenogin VA. Development and applications of Lusternik-Vishik's asymptotic method. Uspekhi mathematicheskikh nauk. 1970; 4(154):123-156.

[3] Demidov AS. Vishik-Lusternik's method and two problems of magnetic hydrodynamics on plasm and tokamak. Probl. math. anal. 2013;(69):5-22.

[4] Kirpikova OI, Roshenkina NA. Small parameter in parabolic and elliptic problems. Mathematics. Education: Proceedings of the 21-th International Conference, Cheboksary. $2013 ; 362$.

[5] Tikhonov AN. On dependence of solutions of differential equations on small parameter. Matem. Sb. 1948; 22 (64):193-204.

[6] Pontryagin LS. Asymptotic behavior of solutions of system of differential equations with small parameter under higher derivatives. Izv. AN SSR, ser. math. 1957;21(5):605-626.

[7] Vishik MI, Lusternik LA. Regular degeneration and boundary layer for linear differential equations with a small parameter. Uspekhi Matematicheskikh Nauk. 1957;12(5): 3-122.

[8] Vishik MI, Lusternik LA. Solution of some perturbation problem in the case of matrices and self-adjoint and not self-adjoint differential equations. Uspekhi Matematicheskikh nauk. $1960 ; 15(3): 3-80$. 
[9] Vazov V. Asymptotic expansion of solutions of ordinary differential equations. Nauka, Moscow; 1968.

[10] Lomov JA. Introduction to general theory of singular perturbations. Nauka, Moscow; 1981.

[11] Ilin A.M. Concordance of asymptotic expansions of solutions of boundary value problems. Nauka, Moscow; 1989.

[12] Javadov MG, Sabzaliev MM. On a boundary value problem for one-characteristic equation degenerating in to one-characteristic one. DAN SSSR. 1979;245(5):1041-1046.

[13] Javadov MG, Sabzaliev MM. On a boundary value problem for singularly perturbed onecharacteristic degeneratial equation. Manscript was deposited in Az NIINTI. 1988;958 - Az.

[14] Javadov MG, Sabzaliev MM. On a boundary value problem for one-characteristic degenerating into elliptic one. Uspekhi Mat. Nauk. 1979;34(4):172.

[15] Sabzaliev MM, Kerimova ME. Asymptotics of the solution in a rectangle of a boundary value problem for one-characteristic differential equation degenerating into a parabolic equation. Transactions of NAS of Azerbaijan, iss. Math. Mech. 2014;34(4):97-106.

[16] Sabzaliev MM, Kerimova ME. Asymptotics of the solution in an infinite semi-strip for one characteristic differential equation degenerating into a parabolic equation. Nonlinear Analysis and Differential Equations. 2016;4(4):179-187.

[17] Sabzaliev MM, Kerimova ME. Asymptotics of the solution of a boundary value problem for one - characteristic differential equation degenerating into a parabolic equation in a infinite strip. Nonlinear Analysis and Differential Equations. 2014;2(3):125-133.

[18] Salimov Ya. Sh, Sabzalieva IM. On asymptotics of the solution of a boundary value problem for odd order singularly perturbed equation. Izvestia AN Azer. SSR. Ser. Techn. I Math. Sci. 1998;5:11-14.

[19] Sabzalieva IM. Asymptotics of solution of a boundary value problem stated on an infinite strip for non-classic type singular perturbed equation. News of Pedagogical University. Ser. of natural sciences, Baku. 1999;1:35-41.

[20] Sabzalieva IM. Boundary value problems for a class of nonclassic type parabolic degenerating equation. Baku. 2004;1:39-49.

[21] Salimov Ya. Sh, Sabzalieva IM. On boundary value problems for a class of non-classical type singularly perturbed differential equations. Doklady Rossiyskoi Akademik Nauk. 2004;399(4):450-456.

[22] Salimov Ya. Sh, Sabzalieva IM. On boundary value problems for a class of singularly perturbed equations of arbitrary odd order. Different. Uravn. 2006;42(5):653-659.

[23] Vishik MI, Listernik LA. On asymptotics of solutions of boundary value problems for quasilinear differential equations. DAN SSSR. 1958;121(5):778-781.

[24] Yui Chen Su. Asymptotics of solutions of some degenerating quasilinear hyperbolic equations of second order. 1961;138(1):935-938.

[25] Trenogin VA. On asymptotics of solution of almost linear parabolic equations with parabolic boundary layer. Uspekhi Mathematiceskikh Nauk. 1961;16(1):163-169.

[26] Lunin V. Yu. On asymptotics of solution of the first boundary value problem for quasilinear elliptic equations of second order. Vestnik Moskovskogo Universiteta, Ser. math. Mech. 1976 ;3:43-51.

[27] Del pino Manuel, Felmer Particiol. Localizing spikelayer patterns singularly perturbed elliptic problems. Tohoku Math. Publ. 1988;8:35-42.

[28] Hale Jack K. Salazar domingo. Tohoku Math. J. 1999;51(3):421-432. 
[29] Misoguchi Norico, Yunagida Eiji. Life span of solutions for a semilinear parabolic problem with small diffusin. J. Math. anat and Appl. 2001;261(1):350-368.

[30] Khanayev MM. Constructing the solution of ill-posed singularly perturbed problem for equation with nonlinearity. Diff. Uravn. 2004;40(6):818-819.

[31] Denisov IV. Angular boundary layer in non-monotone singularly perturbed boundary value problems with nonlinearities. Zh. Vychise. Matem. I Matem. Fiziki. 2004;44(9):1674-1692.

[32] Byeon Jaeyoung. Singularly perturbed nonlinear Dirichlet problems with a general nonlinearity. Trans. Amer. Math. Soc. 2010;362(9):1981-2001.

[33] Sabzaliev MM. On asymptotics of solution of boundary value problem for a quasilinear elliptic equation on infinite strip. Proceedings of Inst. of Mathematics and Mechanics of NAS of Azerbaijan. 2009;31:145-156.

[34] Sabzaliev MM. On asymptotics of the solution of a boundary value problem for singularly perturbed quasilinear elliptic equation in semi-infinite strip. Proceedings of Inst. of Mathematics and Mechanics of NAS of Azerbaijan. 2010;32:175-188.

[35] Sabzaliev MM. On asymptotics of solution to a boundary value problem for a quasilinear hyperbolic equation in an infinite strip. Transactions of NAS of Azerbaijan Iss. Math. Mech. 2010;30(4):159-170.

[36] Sabzaliev MM. Asymptotics of the solution of mixed problem for a quasilinear hyperbolic equation degenerating into parabolic equation. Proceedings of Inst. of Mathematics and Mechanics of NAS of Azerbaijan. 2011;31:79-90.

[37] Sabzaliev MM. Asymptotics of the solution of a boundary value problem for a quasilinear equation in a curvilinear trapezoid. Docl. Rossiyskoy Akademii Nauk. 2012;444(4):1-4.

[38] Sabzaliev MM. On a boundary value problem for a quasilinear elliptic equation degenerating into a parabolic equation in an infinite strip. Nonl. Analisis and Differential equations. 2013; 1(1):1-14.

[39] Sabzaliev MM. The asymptotic form of solution of the boundary value problem for singularly perturbed quasilinear parabolic differential equation. Proceedings of inst. of Math. and Mech. of Nas of Azerbaijan. 2004;21:169-176.

(C) 2017 Sabzaliev and Sabzalieva; This is an Open Access article distributed under the terms of the Creative Commons Attribution License (http://creativecommons.org/licenses/by/4.0), which permits unrestricted use, distribution, and reproduction in any medium, provided the original work is properly cited.

Peer-review history:

The peer review history for this paper can be accessed here (Please copy paste the total link in your browser address bar)

http://sciencedomain.org/review-history/19309 